\title{
Extension System of a Field of View for People Losing Sight in One Eye Using Content-Aware Image Resizing
}

\author{
Hideaki Orii ${ }^{a, *}$, Shohei Hasegawa ${ }^{b}$, Hideaki Kawano ${ }^{b}$, Hiroshi Maeda ${ }^{b}$, Takaharu Kouda ${ }^{a}$ \\ a Fukuoka University, 8-19-1 Nanakuma, Jonan-ku, Fukuoka 814-0180, JAPAN \\ b Kyushu Institute of Technology, 1-1 Sensui-cho, Tobata-ku, Kitakyushu 804-8550, JAPAN \\ *Corresponding Author: oriih @ fukuoka-u.ac.jp
}

\begin{abstract}
People losing sight in one eye as the cause of a disease or trauma have a blind spot for the field of view. They have a lot of accidental situation in daily life, because it could be impossible to recognize the potential hazards which are present in the blind spot. In this paper, we propose an extension system of a field of view for people losing sight in one eye. In the proposed system, the full field of view information of user is captured by wearable camera, and the visual information is compressed and displayed for user with head-mounted camera. In the experiments, we apply the proposed method to actual seen images in daily life. The experimental results show the effectiveness of proposed method.
\end{abstract}

Keywords: image transformation, image resizing, vision aids

\section{Introduction}

People losing sight in one eye as the cause of a disease or trauma have a blind spot for the field of view. It is said that a normal person have a field of view that is about 60 degrees in the upper and about 70 degrees lower in the vertical direction, and about 180 degrees to 200 degrees in the horizontal direction. Since horizontal direction of the field of view of one eye is about 120 degrees, the people losing sight in one eye are missing the field of view of about 60 to 80 degrees than the full field of view. They have a lot of accidental situation in daily life, because it could be impossible to recognize the potential hazards which are present in the blind spot. Fig. 1 shows the comparison of a field of view of sighted people and the people losing partial vision. A car is unrecognized in left

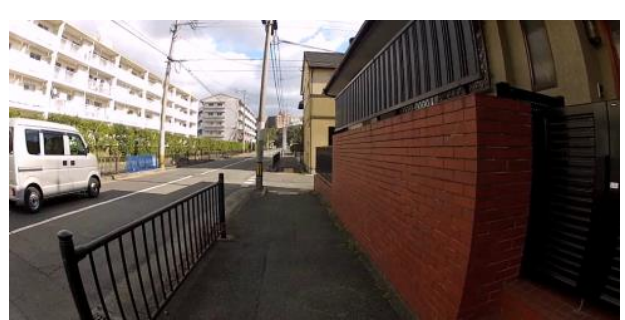

(a) A full field of view.

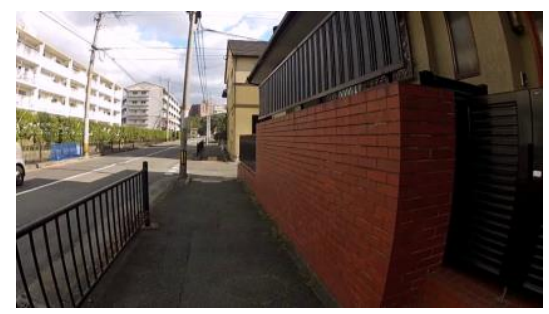

(b) A simulation vision of people losing sight in one eye.

Fig. 1. Comparison of a field of view.

side in Fig. 1 (b). The people losing partial vision has to live with inconvenience, in a lot of situations e.g. climbing stairs, walking the street, reading information including route maps and signs at public places, such as stations and airports.

To solve these problems, the daily life support systems by wearable computing is studied and developed. Various research and development has been proceeding because of minimization of component and performance improvement in recent years. For example, Bryant ${ }^{(1)}$ developed the wearable system for low vision. The system detects obstacles adjacent to user with infrared radiation. And location information is presented to the fiber retinal scanning display. Toyoura et al. proposed ${ }^{(2)}$ a mono-glass 


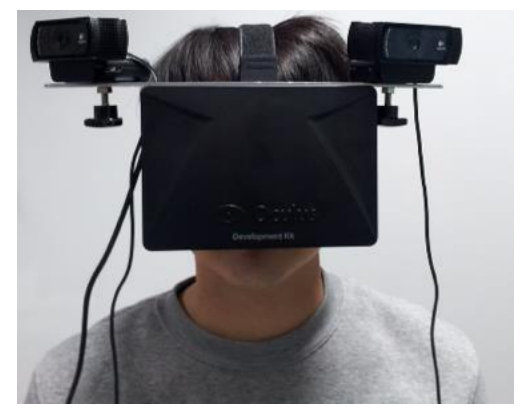

Fig. 2. Proposed system.

system for providing distance information for people losing sight in one eye. The proposed mono-glass is a wearable device with two cameras and one display. The two cameras capture the images on behalf of user's eyes. Depth information is then reconstructed from the captured images and visualized with defocusing for the healthy eye. We have proposed wearable vision aid system employing eye expression recognition ${ }^{(3)}$. This system recognizes user's act of squinting by using a wearable camera, and displays the scaled view image to the user. Those are the approaches of increasing the visibility of the user by presenting enlarged visual information.

In this paper, we propose an extension system of a field of view for people losing sight in one eye. In the proposed system, the full field of view information of user is captured by wearable camera, and the visual information is compressed and displayed for user with head-mounted camera.

\section{Proposed Method}

The proposed system is composed of two cameras and one display as shown in Fig. 2. In proposed system, the blind spot of the user's field of view are captured by head-mounted camera, and the summary image of blind spot is generated by content-aware image resizing technique. Section 2.1 describes the overview of proposed system and Section 2.2 describes proposed method of content-aware image resizing for summarize of visual information.

\subsection{Overview of Proposed System}

In proposed system, the visual information captured from two cameras is summarized and displayed for user. Fig. 3 shows the concept of proposed method. The flow of image processing is shown in below.

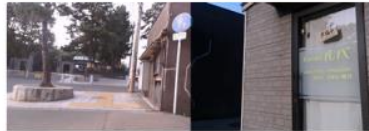

Full field of view

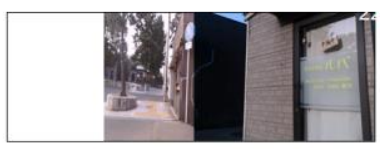

Summarization of Visual Information

Without changing the center point of view, the visual information is summarized

Fig. 3. Concept of Proposed system.

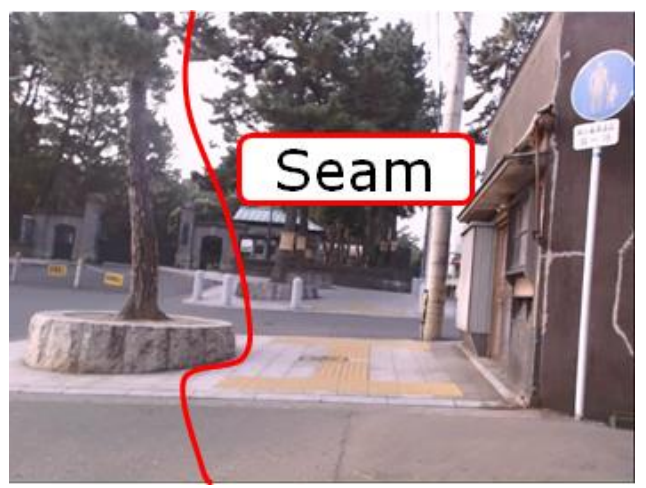

Fig. 4. Example of "Seam".

(1) Capture vision image of user with two head-mounted cameras

(2) Obtain full field of view image by synthesizing of the two images

(3) Summarize full field of view image for losing sight in one eye

(4) Display for user by head-mounted display

In proposed method, to prevent user's confusion, the full field of view image is resized for losing sight in one eye without changing the center point of view. Thus, the half of image is only resizing, and necessary visual information should be remained in result image, such as the potential hazards which are present in the blind spot. The detail of resizing algorithm is described next Section.

\subsection{Content-Aware Image Resizing for Summarize of Visual Information}

To remain the necessary visual information in resizing, we employ content aware image resizing technique ${ }^{(4)}$. In this technique, all pixels in an image are weighted based on image feature, such as a gradient magnitude, and eliminate low cost seams that are line composed of pixels sequentially. The example of "seam" is shown in Fig. 4. The flow of this 


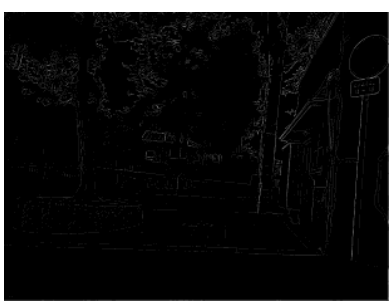

(a) Laplacian edge map,

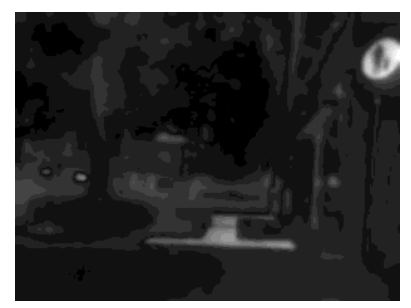

(b) Saliency map.
Fig. 5. Energy maps.

process is performed as following procedure:

(1) Calculate the energy map based on image feature, e.g. absolute value of the Laplacian of the input image.

(2) Calculate "seam" as the low cost path from top end to lower end of image (energy map)

(3) Eliminate the seam pixels

(4) Repeat (2), (3) until satisfying a requirement of image width

To detect the necessary visual information, such as the potential hazards which are present in the blind spot, we employ laplacian edge map and saliency map ${ }^{(5)}$ as energy map. Fig. 5 shows the energy maps obtained by these methods.

\section{Experimental Results and Discussions}

In this section, we apply the proposed method to the actual seen images in daily life. The experiment is a simulation to verify the proposed image resizing algorithm's effectiveness, therefore we implement the algorithm on a personal computer and perform offline experiment with actual data. The PC with implementation was OS: Windows 7 Professional, CPU: Intel Core i5 $3.2 \mathrm{GHz}$, RAM: $8 \mathrm{~GB}$, and the calculation time is a few seconds per 1 frame with respect to VGA size image.

Fig. 6 and 7 show the comparisons of a field of view of images obtained by proposed method. In Fig. 6 (b) and Fig. 7 (b), the objects in image crumple up, because of simple resizing. In Fig. 6 (c), (d), and Fig. 7 (c), (d), despite left half of images are resized, the visibility of the object is not decreased. Additionally, a signage in result image of proposed method is more visible than other results.

\section{Conclusions}

In this paper, we proposed an extension system of a field of view for people losing sight in one eye. In the proposed system, the full field of view information of user is captured by wearable camera, and the visual information is compressed and displayed for user with head-mounted camera. In the experiment, we apply the proposed method to the actual seen images in daily life. The proposed method shows superior performance compared with simple resizing method.

The calculation cost of proposed image resizing algorithm is not low, i.e. the calculation time is a few seconds per 1 frame with respect to VGA size image in the experiment. The main cause of this is that the calculation cost of saliency map is high. We are aiming to reduce the calculation cost by employing time-series processing.

In the future, People losing sight in one eye are participated in the experiment, we will make a detailed study of this. A gaze is needed for identify the target to see.

\section{References}

(1) T R.C. Bryant, C.M. Lee, R.A. Burstein, and E.J. Seibel : "Engineering a Low-cost Wearable Low Vision aid Based on Retinal Light Scanning," In Proc. SID 2004, pp.23-28, 2004

(2) M. Toyoura, K. Kashiwagi, A. Sugiura, X. Mao : "Mono-glass for Providing Distance Information for People Losing Sight in One Eye," ACM SIGGRAPH International Conference on Virtual-Reality Continuum and its Applications in Industry (VRCAI), pp.39-42, 2012

(3) H. Orii, M. Aoki, H. Kawano, H. Maeda : "Eye Expression Recognition of Wearable View Magnification Interface for Low Vision," The 1st International Conference on Industrial Application Engineering 2013 (ICIAE2013), pp.194-197, 2013

(4) S. Avidan, A. Shamir, "Seam Carving for Content-Aware Image Resizing," ACM Trans. on Graphics, Vol.26, Issue 3, No.10, 2007

(5) L. Itti, C. Koch, E. Niebur : "A Model of Saliency-Based Visual Attention for Rapid Scene Analysis," IEEE Transactions on Pattern Analysis and Machine Intelligence, Vol.20, No.11, pp.1254-1259, 1998 


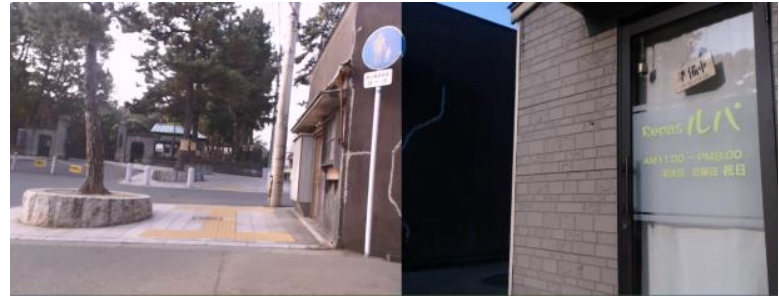

(a) Input image.

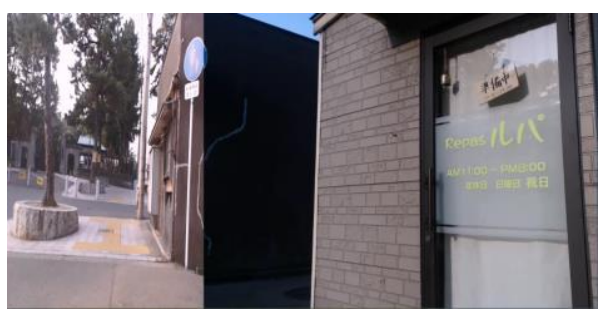

(b) Simple resized image.

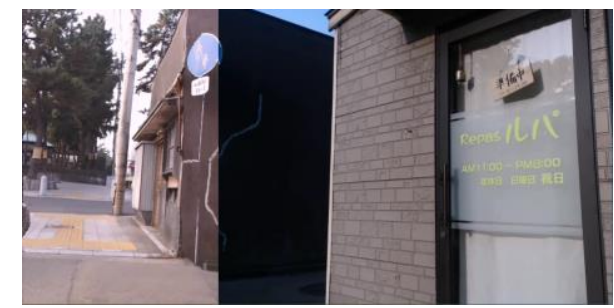

(c) Resized image by method ${ }^{(4)}$.

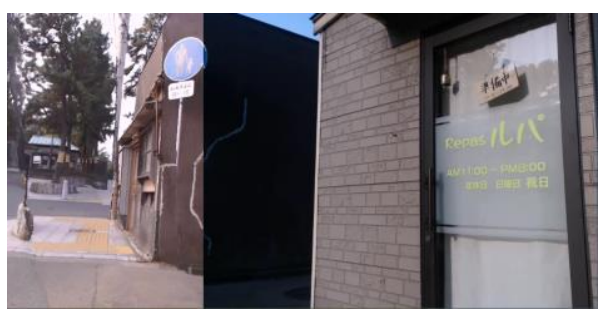

(d) Resized image by proposed method.

Fig.6 Comparison of a field of view of images.

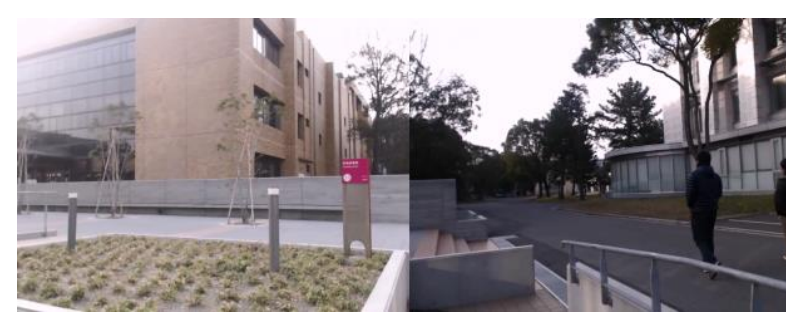

(a) Input image.

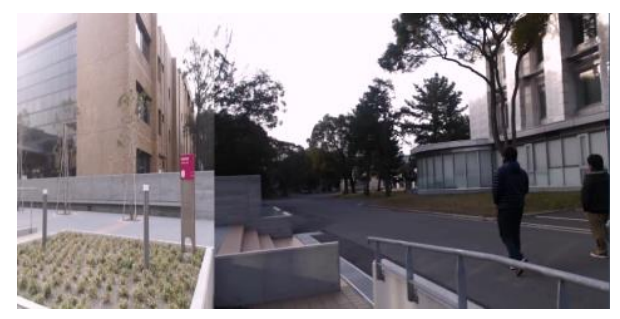

(b) Simple resized image.

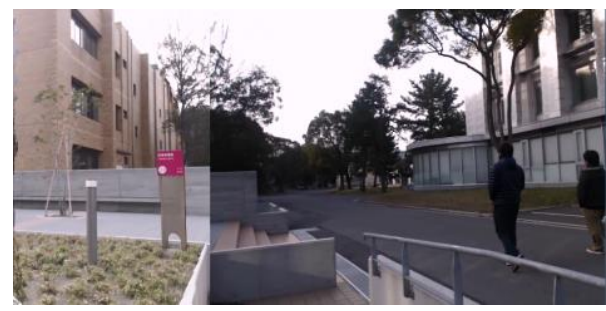

(c) Resized image by method ${ }^{(4)}$.

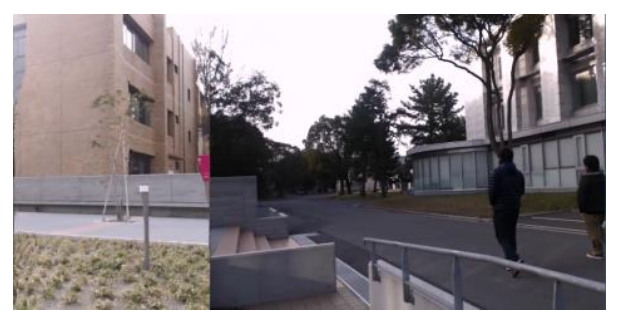

(d) Resized image by proposed method.

Fig.7 Comparison of a field of view of images. 